\title{
Set of Perfect Reconstruction Non-uniform Filter Banks via a Tree Structure
}

\author{
Wing-kuen Ling and P. K. S. Tam \\ Department of Electronic and Information Engineering \\ The Hong Kong Polytechnic University \\ Hung Hom, Kowloon, Hong Kong \\ Hong Kong Special Administrative Region, China \\ Tel: (852) 2766-6238, Fax: (852) 2362-8439 \\ bingodencserver.eie.polyu.edu.hk
}

\begin{abstract}
In this paper, we propose a novel method to test if a non-uniform filter bank can achieve perfect reconstruction via a tree structure. The set of decimators is first sorted in an ascending order. A non-uniform filter bank can achieve perfect reconstruction via a tree structure if and only if some or all of the channels corresponding to the maximum decimation ratio can be grouped into one channel, and the procedure can be repeated until all the channels are grouped together.
\end{abstract}

\section{Introduction}

Non-uniform filter banks play an important role in this decade and they are widely applied to digital image compression $[3,6,7,9,13]$. By realizing a nonuniform filter bank via a tree structure [1,2, 4, 5, 10-12], the filter length in the filters is reduced, improving the computation complexity and the implementation speed [14]. However, not all the non-uniform filter banks can be realized via a tree structure $[5,8$, 10-12]. A method to compute the number of combinations of sub-trees is proposed [8] to test if the decimators in the non-uniform filter bank can be generated by a tree structure. However, even though the decimators can be generated by a tree structure, this does not imply that the non-uniform filter bank can be generated by a tree structure. This is because the analysis filters are ignored in the consideration. In this paper, the necessary and sufficient conditions for a non-uniform filter bank to be realized by a tree structure are addressed.

The necessary and sufficient conditions are discussed in section 2 and illustrative examples are presented in section 3. Finally, a conclusion is given in section 4 . 


\section{Necessary and Sufficient Conditions for Realizing a Non- uniform Filter Bank via a Tree Structure}

Let the ordered set of decimators $\left\{n_{0}, \ldots, n_{0}, \ldots, n_{N-1}, \ldots, n_{N-1}\right\}$ be $\mathbf{D}$, where $n_{i}>n_{j}$ for $i>j$, and the multiplicity of $n_{i}$ in $\mathbf{D}$ be $p_{i}$. Let the corresponding analysis filters and synthesis filters be $\left\{H_{0,0}(z), \cdots, H_{0, p_{0}-1}(z), \cdots, H_{N-1,0}(z), \cdots, H_{N-1, p_{N-1}-1}(z)\right\} \quad$ and $\left\{G_{0,0}(z), \cdots, G_{0, p_{0}-1}(z), \cdots, G_{N-1,0}(z), \cdots, G_{N-1, p_{N-1}-1}(z)\right\}$, respectively.

If there exists a set of filters $\left\{H_{N-1}^{\prime}(z), H_{N-1, k_{0}}^{\prime}(z), \cdots, H_{N-1, k_{K_{N-1}-1}}^{\prime}(z)\right\}$, where $k_{i} \in\left[0 p_{N-}\right.$ $\left.{ }_{1}-1\right]$ for $i=0,1, \ldots, K_{N-1}-1$ and $K_{N-1} \in\left[2 p_{N-1}\right]$, such that:

$$
\begin{gathered}
n_{N-1} / K_{N-1} \in \mathrm{Z}, \\
H_{N-1}^{\prime}(z) \cdot H_{N-1, k_{i}}^{\prime}\left(z^{\frac{n_{N-1}}{K_{N-1}}}\right)=H_{N-1, k_{i}}(z)
\end{gathered}
$$

and

$$
\operatorname{det}\left(\left[\begin{array}{cccc}
H_{N-1, k_{0}}^{\prime}(z) & H_{N-1, k_{1}}^{\prime}(z) & \cdots & H_{N-1, k_{K_{N-1}-1}}^{\prime}(z) \\
H_{N-1, k_{0}}^{\prime}\left(z \cdot W_{N-1}\right) & H_{N-1, k_{1}}^{\prime}\left(z \cdot W_{N-1}\right) & \cdots & H_{N-1, k_{N-1-1}}^{\prime}\left(z \cdot W_{N-1}\right) \\
\vdots & \vdots & \ddots & \vdots \\
H_{N-1, k_{0}}^{\prime}\left(z \cdot W_{N-1} K_{N-1}-1\right) & H_{N-1, k_{1}}^{\prime}\left(z \cdot W_{N-1} K_{N-1}-1\right) & \cdots & H_{N-1, k_{K_{N-1}-1}}^{\prime}\left(z \cdot W_{N-1} K_{N-1}-1\right)
\end{array}\right]\right) \neq 0,
$$

where $W_{N-1}=e^{-\frac{j \cdot 2 \cdot \pi}{K_{N-1}}}$, then by a proper design of the synthesis filters, those $K_{N-1}$ channels can be grouped together into one channel with the analysis filter $H_{N-1}^{\prime}(z)$ and the decimator $\downarrow n_{N-1} / K_{N-1}$.

Now, we have a new set of decimators and analysis/synthesis filters. Let the new set of decimators $\left\{n_{0}, \ldots, n_{0}, \ldots, n_{N^{\prime}-1}, \ldots, n_{N^{\prime}-1}\right\}$ be $\mathbf{D}$ ' and the multiplicity of $n_{i}$ in $\mathbf{D}^{\prime}$ be $p_{i}{ }_{i}$. Let the corresponding analysis/synthesis filters be $\left\{H^{\text {new }}{ }_{0,0}(z), \cdots, H^{\text {new }}{ }_{0, p_{0}^{\prime}-1}(z), \cdots, H^{\text {new }}{ }_{N^{\prime}-1,0}(z), \cdots, H^{\text {new }}{ }_{N^{\prime}-1, p_{N^{\prime}-1}^{\prime}-1}(z)\right\} \quad$ and $\left\{G^{\text {new }}{ }_{0,0}(z), \cdots, G^{\text {new }}{ }_{0, p_{0}^{\prime}-1}(z), \cdots, G^{\text {new }}{ }_{N^{\prime}-1,0}(z), \cdots, G^{\text {new }}{ }_{N^{\prime}-1, p_{N^{\prime}-1}^{\prime}-1}(z)\right\}$, respectively.

By repeating the above grouping procedure, if all the channels can be grouped together, and eventually only one channel is left, then the non-uniform filter bank can achieve perfect reconstruction via a tree structure.

\section{Theorem 1}

A non-uniform filter bank can achieve perfect reconstruction via a tree structure if and only if all the channels can be grouped together by the above grouping procedure.

Proof:

The if part is proved in the above. Now, let's consider the only if part. Since the non-uniform filter bank can be realized by a tree structure, $\exists n_{i} \in \mathbf{D}$ such that $n_{i}=n_{N \text { - }}$ ${ }_{1} / K_{N-1}, \quad$ and $\quad \mathrm{a}$ set of filters $\left\{H_{N-1}^{\prime}(z), H_{N-1, k_{0}}^{\prime}(z), \cdots, H_{N-1, k_{K_{N-1}-1}}^{\prime}(z)\right\}$ such that $H_{N-1}^{\prime}(z) \cdot H_{N-1, k_{i}}^{\prime}\left(z^{\frac{n_{N-1}}{K_{N-1}}}\right)=H_{N-1, k_{i}}(z)$. But do those filters satisfy equation (3)? Or in other 
words, if some of the analysis filters in a sub-tree are linearly dependent, does there exist a set of synthesis filters such that the whole system still achieves perfect reconstruction?

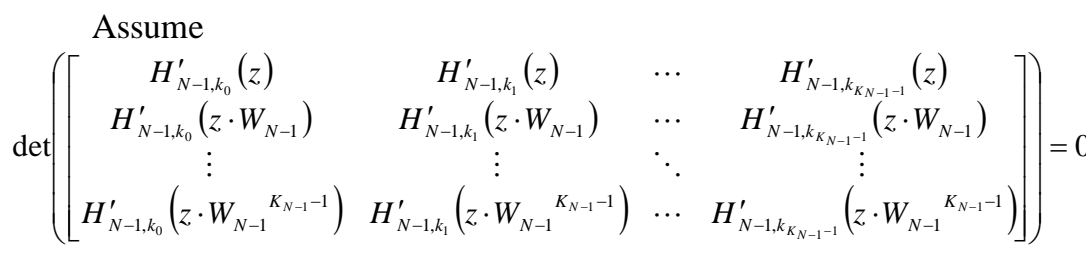
$\exists G_{N-1, k_{0}}(z), \cdots, G_{N-1, k_{N-1-1}}(z)$ and a non-zero transfer function $T(z)$ such that:

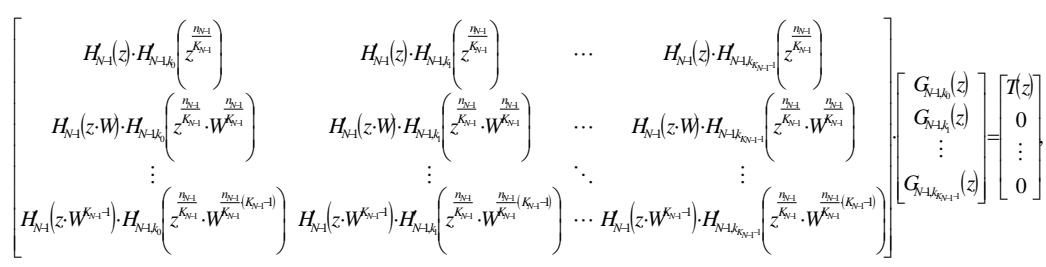

where $W=e^{\frac{j \cdot 2 \cdot \pi}{n_{N-1}}}$.

Since

$\operatorname{det}\left(\left[\begin{array}{cccc}H_{N-1, k_{0}}^{\prime}(z) & H_{N-1, k_{1}}^{\prime}(z) & \cdots & H_{N-1, k_{N-1-1}}^{\prime}(z) \\ H_{N-1, k_{0}}^{\prime}\left(z \cdot W_{N-1}\right) & H_{N-1, k_{1}}^{\prime}\left(z \cdot W_{N-1}\right) & \cdots & H_{N-1, K_{N-1-1}}^{\prime}\left(z \cdot W_{N-1}\right) \\ \vdots & \vdots & \ddots & \vdots \\ H_{N-1, k_{0}}^{\prime}\left(z \cdot W_{N-1}^{K_{N-1}-1}\right) & H_{N-1, k_{1}}^{\prime}\left(z \cdot W_{N-1}^{K_{N-1}-1}\right) & \cdots & H_{N-1, k_{N-1-1}}^{\prime}\left(z \cdot W_{N-1} K_{N-1}-1\right)\end{array}\right]\right)=0 \quad, \quad$ by letting $z=z^{\frac{n_{N-1}}{K_{N-1}}}$, we have:

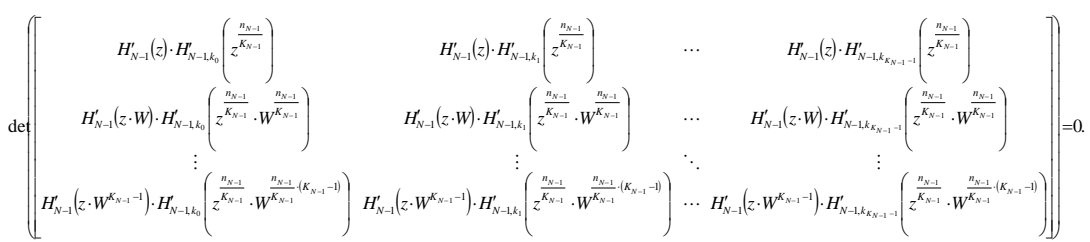

Let the matrix in equation (5) be $\mathbf{H}$. By examining equation (5) and applying Cramer's rule to equation (4), we find that the determinants of the matrices by deleting the first row and any columns are zero. By the modulation principle, we find that the determinants of the matrices by deleting the last row and any columns are zero. Let the rank of the matrix by deleting the first row of $\mathbf{H}$ be $r$, and that of the matrix by keeping the first $r+1$ rows of $\mathbf{H}$ be $H^{\prime}=\left[\begin{array}{lll}h_{0}^{\prime} & \cdots & h_{K_{N-1}-1}^{\prime}\end{array}\right]=\left[\begin{array}{ll}h_{0,0} & h_{0,1} \\ h_{S, 0} & h_{S, 1}\end{array}\right]$, where $\mathbf{h}_{\mathbf{i}}$ is the $i^{\text {th }}$ column of $\mathbf{H}^{\prime}$ and $\mathbf{h}_{\mathbf{0}, \mathbf{0}}$ are the first $r$ elements of the first row of $\mathbf{H}^{\prime}$. Since $H^{\prime} \cdot\left[\begin{array}{l}g_{a} \\ g_{b}\end{array}\right]=\left[\begin{array}{c}T(z) \\ 0 \\ \vdots \\ 0\end{array}\right]$, where $\mathbf{g}_{\mathbf{a}}$ is a vector containing the first $r$ synthesis filters, we have 
$\mathbf{h}_{\mathbf{0 , 0}} \cdot \mathbf{g}_{\mathrm{a}}+\mathbf{h}_{\mathbf{0 , 1}} \cdot \mathbf{g}_{\mathrm{b}}=T(z)$ and $\mathbf{h}_{\mathbf{S}, \mathbf{0}} \cdot \mathbf{g}_{\mathrm{a}}+\mathbf{h}_{\mathbf{S}, \mathbf{1}} \cdot \mathbf{g}_{\mathrm{b}}=\mathbf{0}$. This implies that $\left(\mathbf{h}_{\mathbf{0 , 1},}-\mathbf{h}_{\mathbf{0 , 0} \mathbf{0}} \cdot \mathbf{h}_{\mathbf{S}, \mathbf{0}}{ }^{-\mathbf{1}} \cdot \mathbf{h}_{\mathbf{S}, \mathbf{1}}\right) \cdot \mathbf{g}_{\mathrm{b}}=T(z)$, and

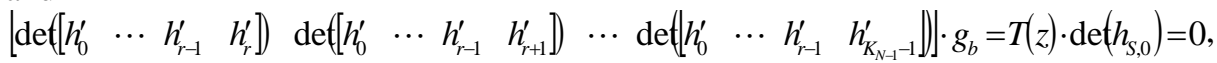
which contradicts the assumption. Hence, if some of the analysis filters in a sub-tree are linearly dependent, there does not exist a set of synthesis filters such that the whole system achieves perfect reconstruction. This proves the only if part and the theorem.

\section{Illustrative Examples}

\subsection{Perfect Reconstruction Tree Structure Filter Bank}

Consider the non-uniform filter bank shown in figure 1:

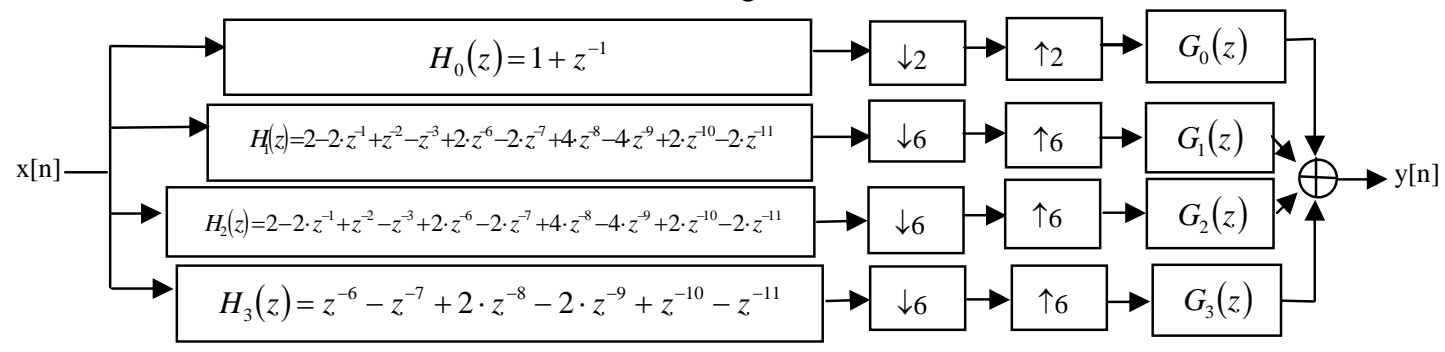

Fig. 1. Perfect Reconstruction tree structure filter bank

In this case, $n_{0}=2, n_{1}=6, p_{0}=1, p_{1}=3$ and $N=2$. By selecting $K_{l}=3, H_{1}^{\prime}(z)=1-z^{-1}$, $H_{1,0}^{\prime}(z)=5+2 \cdot z^{-1}+z^{-3}+2 \cdot z^{-4}+z^{-5} \quad, \quad H_{1,1}^{\prime}(z)=2+z^{-1}+2 \cdot z^{-3}+4 \cdot z^{-4}+2 \cdot z^{-5} \quad, \quad$ and $H_{1,2}^{\prime}(z)=z^{-3}+2 \cdot z^{-4}+z^{-5}[14]$, we can group the last three channels together into one channel with the new analysis filter $H_{1}^{\prime}(z)=1-z^{-1}$ and the decimator $\downarrow 2$. Similarly, by selecting $K_{0}=2, H^{\prime}{ }_{0}(z)=1, H^{\prime}{ }_{0,0}(z)=1+z^{-1}$, and $H^{\prime}{ }_{0,1}(z)=1-z^{-1}$, this non-uniform filter bank can achieve perfect reconstruction via a tree structure.

\subsection{Not Perfect Reconstruction Tree Structure Filter Bank Due to the Dependent Kernel}

Consider the same non-uniform filter bank shown in figure 1 with $H_{0}(z)$ is changed to $F(z) \cdot\left(1-z^{-1}\right)$, where $F(z)=F(-z)$. The last three channels are grouped together with the same procedure as above, and we have two channels left with decimator $\downarrow 2$, and the analysis filters are $F(z) \cdot\left(1-z^{-1}\right)$ and $\left(1-z^{-1}\right)$, respectively. Since 
$\operatorname{det}\left(\left[\begin{array}{cc}H_{0}(z) & 1-z^{-1} \\ H_{0}(-z) & 1+z^{-1}\end{array}\right]\right)=0$, we conclude that this non-uniform filter bank cannot achieve perfect reconstruction even through it can be realized via a tree structure.

\subsection{Cannot Be Realized Via a Tree Structure Filter Bank Due to Structural Problem}

Consider the same non-uniform filter bank shown in figure 1 with $H_{l}(z)$ changed to $\left(1-z^{-1}\right) \cdot F_{1}(z), H_{2}(z)$ changed to $\left(1-z^{-1}\right) \cdot F_{2}(z), H_{3}(z)$ changed to $\left(1-z^{-1}\right) \cdot F_{3}(z)$, where $F_{1}(z) / F_{2}(z)$ and $F_{2}(z) / F_{3}(z)$ are not rational functions of $z^{2}$. In this case, the last three channels cannot be grouped together. Hence, this non-uniform filter bank cannot be realized via a tree structure.

\subsection{Incompatible non-uniform Filter Bank}

Consider an incompatible non-uniform filter bank [15] with the set of decimators $\{2,3,6\}$. Since $p_{i}=1, \forall i$, there does not exist $K_{j} \in\left[2 p_{j}\right]$. Hence, an incompatible non-uniform filter bank cannot be realized via a tree structure [15].

\subsection{Compatible Non-uniform Filter Bank, But Cannot Be Realized Via a Tree Structure}

Consider a non-uniform filter bank with the set of decimators $\{5,5,5,7,7,35,35,35,35\}$. In this case, $n_{0}=5, n_{1}=7, n_{2}=35, p_{0}=3, p_{1}=2, p_{2}=4$, and $N=3$. Since there does not exist $K_{2} \in\left[2 p_{2}\right]$ such that $n_{2} / K_{2} \in \mathbf{Z}$, this non-uniform filter bank cannot be realized via a tree structure.

\section{Conclusion}

In this paper, we propose a novel method to test if a non-uniform filter bank can achieve perfect reconstruction via a tree structure. The advantage of realizing a nonuniform filter bank via a tree structure is to reduce the computation complexity and provide fast implementation for non-uniform filter bank [14].

\section{Acknowledgement}

The work described in this paper was substantially supported by a grant from the Hong Kong Polytechnic University with account number G-V968. 


\section{References}

1. Vaidyanathan P. P.: Lossless Systems in Wavelet Transforms. IEEE International Symposium on Circuits and Systems, ISCAS, Vol. 1. (1991) 116-119.

2. Soman A. K. and Vaidyanathan P. P.: Paraunitary Filter Banks and Wavelet Packets. IEEE International Conference on Acoustics, Speech, and Signal Processing, ICASSP, Vol. 4. (1992) 397-400.

3. Bamberger R. H., Eddins S. L. and Nuri V.: Generalizing Symmetric Extension: Multiple Nonuniform Channels and Multidimensional Nonseparable IIR Filter Banks. IEEE International Symposium on Circuits and Systems, ISCAS, Vol. 2. (1992) 991-994.

4. Sodagar I., Nayebi K. and Barnwell T. P.: A Class of Time-Varying Wavelet Transforms. IEEE International Conference on Acoustics, Speech, and Signal Processing, ICASSP, Vol. 3. (1993) 201-204.

5. Soman A. K. and Vaidyanathan P. P.: On Orthonormal Wavelets and Paraunitary Filter Banks. IEEE Transactions on Signal Processing, Vol. 41, No. 3. (1993) 1170-1183.

6. Vaidyanathan P. P.: Orthonormal and Biorthonormal Filter Banks as Convolvers, and Convolutional Coding Gain. IEEE Transactions on Signal Processing, Vol. 41, No. 6. (1993) 2110-2130.

7. Soman A. K. and Vaidyanathan P. P.: Coding Gain in Paraunitary Analysis/Synthesis Systems. IEEE Transactions on Signal Processing, Vol. 41, No. 5. (1993) 1824-1835.

8. Kovačević J. and Vetterli M.: Perfect Reconstruction Filter Banks with Rational Sampling Factors. IEEE Transactions on Signal Processing, Vol. 41, No. 6. (1993) 2047-2066.

9. Bamberger R. H., Eddins S. L. and Nuri V.: Generalized Symmetric Extension for Size-Limited Multirate Filter Banks. IEEE Transactions on Image Processing, Vol. 3, No. 1. (1994) 82-87.

10. Makur A.: BOT's Based on Nonuniform Filter Banks. IEEE Transactions on Signal Processing, Vol. 44, No. 8. (1996) 1971-1981.

11. Li J., Nguyen T. Q. and Tantaratana S.: A Simple Design Method for NearPerfect-Reconstruction Nonuniform Filter Banks. IEEE Transactions on Signal Processing, Vol. 45, No. 8. (1997) 2105-2109.

12. Akkarakaran S. and Vaidyanathan P. P.: New Results and Open Problems on Nonuniform Filter-Banks. IEEE International Conference on Acoustics, Speech, and Signal Processing, ICASSP, Vol. 3,. (1999) 1501-1504.

13. Omiya N., Nagai T., Ikehara M. and Takahashi S. I.: Organization of Optimal Nonuniform Lapped Biorthogonal Transforms Based on Coding Efficiency. IEEE International Conference on Image Processing, ICIP, Vol. 1. (1999) 624627.

14. Vaidyanathan P. P.: Multirate Systems and Filter Banks. Englewood Cliffs, NJ: Prentice Hall, 1993.

15. Hoang P. Q. and Vaidyanathan P. P.: Non-Uniform Multirate Filter Banks: Theory and Design. IEEE International Symposium on Circuits and Systems, ISCAS, Vol. 1. (1989) 371-374. 